\title{
A Modified Normalized Weighting Factor method for improving the efficiency of the blended high-resolution advection schemes in the context of multiphase flows
}

\author{
Jessica Mariño-Salguero ${ }^{1,2}(\varangle)$, Michael Schäfer ${ }^{1,2}$ \\ 1. Institute of Numerical Methods in Mechanical Engineering (FNB), Technische Universität Darmstadt, Dolivostraße 15, 64293 Darmstadt, \\ Germany \\ 2. Graduate School of Computational Engineering, Technische Universität Darmstadt, Dolivostraße 15, 64293 Darmstadt, Germany
}

\begin{abstract}
This work deals with a new methodology for the implementation of high-resolution (HR) schemes employed to advect the volume fraction in the volume of fluid (VOF) method, in which the numerical stability and convergence depend heavily on the numerical advection scheme and implementation method. The proposed method is based on the normalized weighting factor (NWF) method, which linearizes the normalized interpolation profile and rewrites the face value directly using the donor, acceptor, and upwind nodes. However, unlike the NWF, which is fully implicit and results in pentadiagonal linear systems, the new modified normalized weighting factor (MNWF) method only forms the implicit terms with the contribution of the donor and acceptor nodes, while the contribution of the upwind node explicitly forms part of the source term. Therefore, the method results in a tridiagonal linear system. The comparison of the new method with the deferred correction (DC), downwind weighting factor (DWF), and the RNWF methods shows that the MNWF requires about 5\%-25\% fewer iterations than DC and RNWF, and around $10 \%-85 \%$ less than DWF. Thus, a similar order of accuracy of the results can be obtained with less computational time.
\end{abstract}

Keywords
volume of fluid (VOF)
advection
discretization method
two-phase flow
efficiency
Article History
Received: 28 February 2020
Revised: 19 April 2020
Accepted: 25 April 2020
Research Article
○ The Author(s) 2020

\section{Introduction}

The volume of fluid (VOF) method of Hirt and Nichols (1981) is a well-established conservative method to solve multiphase flow problems. The VOF introduces an additional transport equation to advent a marker function called volume fraction to define the position of the interface between the fluids. The volume fraction must be updated every time that the fluids move, and the boundary between the different fluids changes position. Nevertheless, updating the marker function is critical for the success of the simulation of multiphase flows and also is not a trivial job due to the purely convective nature of the transport equation (Tryggvason et al., 2001).

An option to deal with this problem is using the blended High Resolution (HR) schemes also known as interface-capture schemes which combine a high order $(\mathrm{HO})$ diffusive scheme, a compressive scheme, and the Convection
Boundedness Criterion (CBC) ensuring that no oscillatory behavior is experienced in the solution and have relatively low numerical diffusion (Tryggvason et al., 2001; Moukalled et al., 2016). An example of such schemes is CICSAM (Ubbink and Issa, 1999). However, the direct introduction of the blended HR schemes into the discretized equation is not suitable because of their composite nature. Thus, some techniques initially created to implement the HR schemes in the momentum equation have been used to overcome this difficulty.

For instance, Meyer et al. (2016) implemented the blended interface capture scheme BICS (Wackers et al., 2011) with the deferred correction (DC) method of Rubin and Khosla (1977) to develop a new code for simulating free-surface flows around modern sailing yachts. For the DC method, the implicit terms of the discretized equation are based on the upwind scheme, whereas the difference between the BICS schemes and the upwind scheme is

$\triangle$ marino@fnb.tu-darmstadt.de 


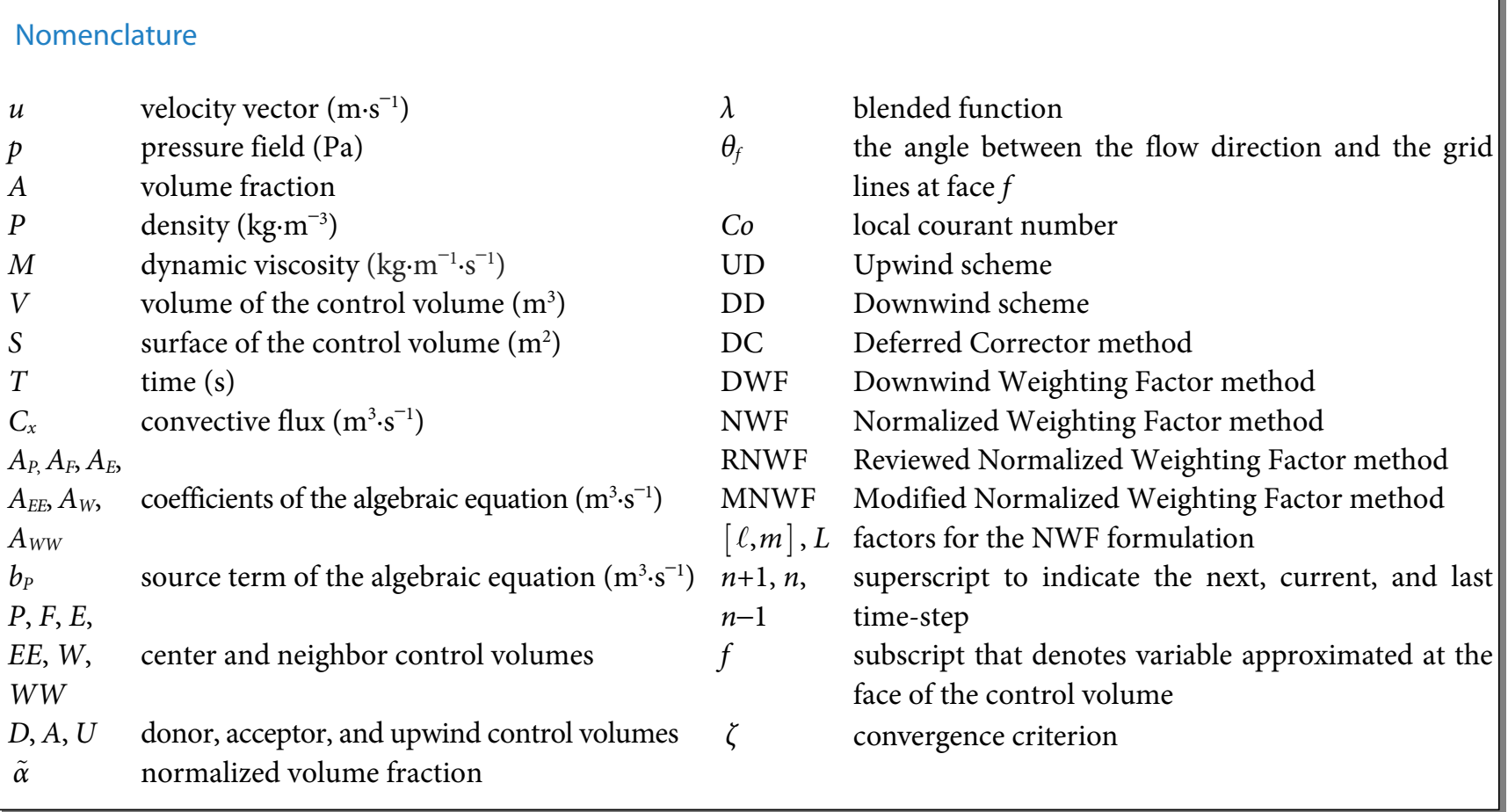

considered as a source term. Although according to Darwish and Moukalled (1996), DC suffers from low convergence rates, whereas the general approach of Meyer et al. showed better performance than other codes. However, the influence of DC on the general approach was not studied.

On the other hand, CICSAM and its modifications such as THOR (Hogg et al., 2006), MCICSAM-W (Wacławczyk et al., 2007), and MCICSAM-Z (Zhang et al., 2014) employ the Downwind Factor Method (DWF) method of Leonard and Mokhtari (1990). The DWF introduces an auxiliary factor that implicitly contains higher-order wide-stencil information, but its implementation involves only the adjacent upwind and downwind node values. So, this method is suitable for tridiagonal solvers. However, the coefficients obtained from a DWF implementation sometimes are not diagonally dominant; thus, the formulation is not stable for many flow configurations and requires substantial relaxation to achieve convergence. Despite the described problem, this method is still used commonly in the multiphase community.

Another technique that overcomes the shortcomings of the DWF method, but which is rarely applied in the context of multiphase flows is the full implicit Normalized Weighing Factor (NWF) method (Darwish and Moukalled, 1996). The NWF linearizes the normalized interpolation profiles and rewrites the face value directly using the central, upwind, and downwind nodes so that the method uses a pentadiagonal stencil, and the diagonal coefficient results are always positive. Consequently, the NWF is much more robust than the DWF and faster than DC methods (Darwish and Moukalled, 1996). Nevertheless, NWF is not frequently used because it requires the pentadiagonal matrix algorithm (PDMA) to solve the system of equations.

In 2018, a revision of the described normalized weighting factor (RNWF) method was presented by Chourushi (2018), which is applicable for tridiagonal equation solvers. This method relies on the final discretization of the normalized weighting factor method and removes the contribution of far-off nodal values from the diagonal coefficient. These terms are later added as a source term. According to the author, the RNWF is four times faster than DC and 1.3 times faster than NWF.

Because of the stability advantages and efficiency of the NWF formulation compared to DC and DWF, and the new possibility of using it with tridiagonal equation solvers, we tested the RNWF method in the context of multiphase fluids and found that the convergence rate of the RNWF is similar to the DC method in the case of multiphase flows and that the RNWF method tends to degenerate the interface slightly. We supposed that the problem lies in the introduction of two explicit terms in the source term, the value of the center point and the upwind point instead of only the upwind point as is suggested in the original NWF method. Our new idea is only to introduce the upwind value as a source term.

This paper presents this new idea that we call Modified Normalized Weighting Factor (MNWF) method which we apply for the numerical implementation of six blended HR schemes: CICSAM, MCICSAM-W, MCICSAM-Z, HRIC (Muzaferija et al., 1998), FBICS (Tsui et al., 2009), and CUIBS (Patel and Natarajan, 2015). The implementations 
are realized on the in-house finite-volume flow solver FASTEST, based on a block-structured collocated grid arrangement. For the investigation, we consider four test cases: the slotted circle, the circle in shear fluid, the rising bubble, and the break-dam with an obstacle. The convergence rate is given by the total number of iterations required for convergence, and the accuracy of the results is analysed for each test case and compared with the obtained one using the DC, DWF, and RNWF methods.

In the following sections, the mathematics and results of the new approach are presented. First, the governing conservation equations of the multiphase dynamics are shown, as well as the discretization process of the volume fraction equation. Then, the DC, DWF, NWF, and RNWF methods are reviewed, and their disadvantages are pointed out. Finally, the modified normalized weighing factor (MNWF) method is described in detail and used to solve the test problems.

\section{Governing equations}

Two viscous, incompressible, and immiscible fluids are modelled as a single effective fluid whose behavior is described by the conservation transport equations of mass, momentum, and volume fraction:

$$
\begin{gathered}
\nabla \cdot u=0 \\
\frac{\partial \rho u}{\partial t}+\rho u \cdot \nabla u+\mu \nabla^{2} u=-\nabla p+\rho g+f_{\sigma} \\
\frac{\partial \alpha}{\partial t}+u \cdot \nabla \alpha=0
\end{gathered}
$$

where $u$ is the velocity vector, $p$ the pressure field, $\alpha$ the volume fraction field of one of the fluids, and $\rho, \mu$ are the physical properties density and dynamic viscosity, respectively. In the momentum equation (Eq. (2)), the last two terms of the right-hand side represent the gravity forces and the force due to surface tension. Since the multiphase system of the two fluids is treated as a single fluid, the local density and dynamic viscosity can be evaluated as a function of the volume fraction using the following relations:

$$
\rho=\alpha \rho_{1}+(1-\alpha) \rho_{2}, \quad \mu=\alpha \mu_{1}+(1-\alpha) \mu_{2}
$$

where the subscripts 1 and 2 refer to each fluid and $\alpha$ denotes the presence $(\alpha=1)$ or absence $(\alpha=0)$ of the traced fluid. A volume fraction between 0 and 1 indicates the presence of a mixture, and the value of 0.5 defines the interface between the fluids.

The three conservation equations are discretized in space with the second-order finite-volume method and in time with the second backward differentiation BDF2 time scheme (Ferziger and Perić, 2012). The discretization of the mass and momentum equations for multiphase flow systems follows the methodology detailed in Sauer (2000) and it is not shown here as it is outside the focus of this paper. However, the following sections will be dedicated to the discretization of the volume fraction transport equation.

\section{Discretization of the volume fraction transport equation}

In order to solve the volume fraction governing equation, the domain is subdivided into an arbitrary number of control volumes which form a structured grid domain. Then, Eq. (3) is integrated over each control volume, and the volume integral of the divergence of $\alpha$ is transformed to a surface integral applying the Gauss theorem. Hence, the integral form of the volume fraction transport equation results in

$$
\frac{\partial}{\partial t} \int_{V} \alpha \mathrm{d} V+\int_{S} \alpha u \cdot n \mathrm{~d} S=0
$$

where $V$ is the volume and $S$ is the surface of the control volume, and $n$ is the normal vector to the surface. The volume integral is resolved using the BDF2 time scheme, and the surface integral is numerically approximated by the mid-point ruler. For an arbitrary control volume $P$ Eq. (5) becomes

$$
\begin{aligned}
\frac{3 \alpha_{P}^{n+1}-4 \alpha_{P}^{n}+\alpha_{P}^{n-1}}{2 \Delta t} \Delta V_{P} & =-\sum_{f \sim n b(P)}(u \alpha)_{f}^{n+1} \cdot S_{f} \\
& =\sum_{f \sim n b(P)} C_{f} \alpha_{f}^{n+1}
\end{aligned}
$$

where the superscripts $n+1, n$, and $n-1$ represented the values at the next, current, and last time-step respectively. The subscript $f$ denotes the variable approximated at the center of each face of the control volume $P$, and $C$ is the convective flux. After some arithmetic operation, the algebraic form of the discretized equation reads

$$
\left(A_{P(\text { time })}+A_{P}\right) \alpha_{P}=\sum_{F \sim N B_{P}} A_{F} \alpha_{F}+b_{P}
$$

with $A_{P(\text { time })}=\frac{3 \Delta V_{P}}{2 \Delta t}$ and $b_{P}=\frac{-4 \alpha_{P}^{n}+\alpha_{P}^{n-1}}{2 \Delta t} \Delta V_{P} . A_{P}$ and $A_{F}$ are defined according to the advection scheme used to approximate the face volume fraction $\alpha_{f}^{n+1}$.

\section{Blended high resolution (HR) schemes}

The accuracy of the numerical solution of Eq. (7) depends on the proper estimation of the face volume fraction. This demands an advection scheme that should neither produce numerical diffusion nor unbounded values (Muzaferija et al., 1998). Over the last decades, blended advection schemes between a compressive and a diffusive high resolution (HR) scheme have been used to advect the volume fraction. The 
reason for this is, that the use of just the compressive schemes can cause an alignment of the fluid interface with the grid (Ubbink, 1997), or that the use of only the diffusive HR schemes deteriorates the accuracy when the flow is not orientated along a grid line due to the false diffusion (Darwish and Moukalled, 2006). The switching strategy depends on the angle $\theta_{f}$ between the flow direction and the grid lines. This approach has been employed to develop several blending advection schemes, also known as interfacecapture schemes, for example, CICSAM (Ubbink and Issa, 1999), HRIC (Muzaferija et al., 1998), STACS (Darwish and Moukalled, 2006), and FBICS (Tsui et al., 2009).

A high resolution scheme is a composite high-order scheme combined with the Convective Boundedness Criterion (CBC) of Gaskell and Lau (1988) to ensure that the interpolation profile at the cell face does not underflow or overflow the cell (Moukalled et al., 2016). Some examples of them are SUPERBEE, MUSCL, SMART, or STOIC. The HR schemes can be formulated in the framework of the Normalized Variable Diagram (NVD) introduced by Leonard (1991), which is illustrated in Fig. 1. The UD line refers to the upwind differencing scheme, DD to the downwind differencing scheme, and the shaded area indicates the part of the NVD that fulfils the CBC. The schemes close to the UD line are linked with numerical diffusion but always produce a bounded solution and are stable, whereas the schemes near the DD line are unstable but introduce a negative numerical diffusion, so they are known as compressive schemes.

For the NVD, the normalized volume fraction $\tilde{\alpha}$ in the vicinity of the cell $P$ is defined as

$$
\tilde{\alpha}(r)=\frac{\alpha(r)-\alpha_{U}}{\alpha_{A}-\alpha_{U}}
$$

where the subscripts $D, A$, and $U$ refer to the donor, acceptor, and upwind cells designated depending on the flux direction (see Fig. 2), and $\boldsymbol{r}$ is the position vector. With this normalization relation $\tilde{\alpha}_{U}=0, \tilde{\alpha}_{A}=1$, and the normalized volume fraction at the cell face $\tilde{\alpha}_{f}$ becomes a function of $\tilde{\alpha}_{D}$.

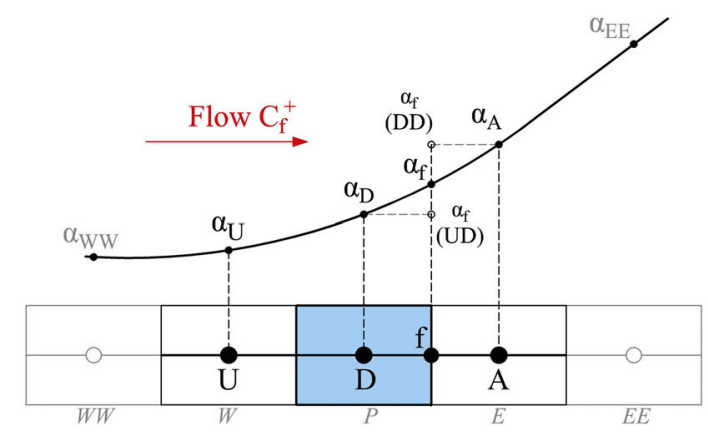

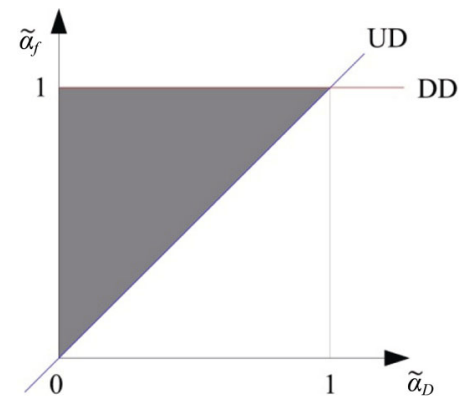

Fig. 1 NVD combined with the CBC showing the region where $\tilde{\alpha}_{f}$ is bounded.

Then, a blended HR scheme designed within the NVD framework defines the normalized face volume fraction as

$$
\tilde{\alpha}_{f(\text { blended HR })}=\lambda \tilde{\alpha}_{f(\text { compressive })}+(1-\lambda) \tilde{\alpha}_{f(\text { HR scheme })}
$$

where the blending function $\lambda=f\left(\theta_{f}\right)$ varies between 0 and 1 .

Due to its composite nature $\tilde{\alpha}_{f(\text { blended HR) }}$ cannot be directly expressed in terms of the nodal values of the control volume $P$ and neighbors $F$, which is necessary to determine the $A_{P}$ and $A_{F}$ coefficients and to solve Eq. (7) for the unknown values at the central nodes. So, several methodologies have been developed for the numerical implementation of the blended HR schemes. The following section provides a brief introduction of some commonly used methods, and the next section explains in detail the proposed new alternative.

\section{Major implementation methods for HR schemes}

\subsection{Deferred corrector approach}

The deferred corrector (DC) method of Rubin and Khosla (1977) is a simple technique designed for tridiagonal matrix solvers that define the convective term of the volume fraction equation as

$$
C_{f} \alpha_{f}=C_{f} \alpha_{f}^{\mathrm{UD}}+C_{f}\left(\alpha_{f}^{\text {blended HR }}-\alpha_{f}^{\mathrm{UD}}\right)
$$

The first term, the value obtained by the upwind scheme, is used to form the coefficient matrix $A$ for the nodal

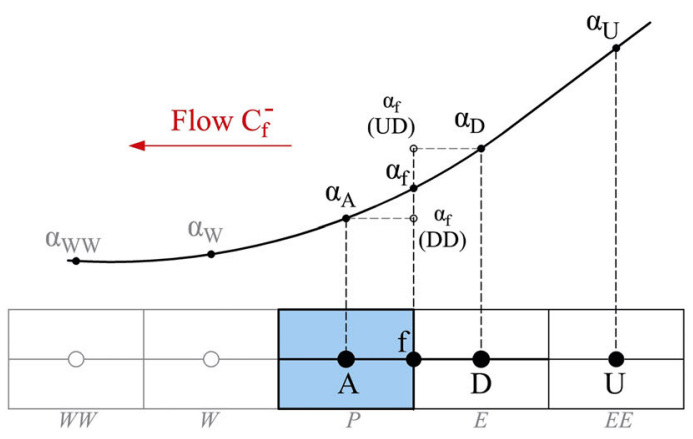

Fig. 2 Schematic position of the nodes $D, A$, and $U$ according to the direction of the flow in a one-dimensional domain. 
algebraic equation, while the second term, the difference between the blended HR and UD schemes is explicitly computed using the last available values and added to the source term. Thus, the resulting coefficient matrix is always diagonally dominant, yielding a numerically stable method. Nevertheless, the convergence rate decreases as the difference between the cell face value estimated by the upwind scheme and the blended HR scheme increases (Moukalled et al., 2016).

\subsection{Downwind Weighting Factor method}

The Downwind Weighing Factor (DWF) method was developed by Leonard and Mokhtari (1990) to overcome the low convergence rate associated with the DC method. The face value is defined as a weighted average between the donor and acceptor cell written as

$$
\alpha_{f}=D W F_{f} \alpha_{A}+\left(1-D W F_{f}\right) \alpha_{D}
$$

where the $D W F$ is the weighting factor that varies between 0 and 1 and is explicitly computed as

$$
D W F_{f}=\frac{\tilde{\alpha}_{f}^{\text {blended } \mathrm{HR}}-\tilde{\alpha}_{D}}{1-\tilde{\alpha}_{D}}
$$

Then, the convective term using nodal values and considering the flow direction takes the form

$$
\begin{aligned}
C_{f} \alpha_{f}= & \max \left(0, C_{f}\right)\left[D W F_{f} \alpha_{F}+\left(1-D W F_{f}\right) \alpha_{P}\right]- \\
& \max \left(-C_{f}, 0\right)\left[D W F_{f} \alpha_{P}+\left(1-D W F_{f}\right) \alpha_{F}\right]
\end{aligned}
$$

which is used to generate the coefficient matrix $A$.

The effect of this weighting formulation is a reduced stencil for the discretized coefficients, which allows the system of equations to be solved with a tridiagonal solver. However, the diagonal coefficient $A_{P}$ becomes negative when $\alpha_{f}>0.5\left(\alpha_{D}+\alpha_{A}\right)$ which is a common scenario for all HR schemes when $\alpha_{D}>0.5$. Consequently, this system of equations leads to unphysical results for many flow configurations and requires substantial relaxation to avoid convergence problems (Darwish and Moukalled, 1996).

\subsection{Normalized Weighting Factor method}

The Normalized Weighting Factor (NWF) method of Darwish and Moukalled (1996) describes the normalized face value as a linear function of the normalized donor value write as

$$
\tilde{\alpha}_{f}=\ell \tilde{\alpha}_{D}+m
$$

where $\ell$ represents the slope and $m$ the intercept of each linear function that is part of the HR scheme employed. Then, this linear relation is rewritten as

$$
\frac{\alpha_{f}-\alpha_{U}}{\alpha_{A}-\alpha_{U}}=\ell \frac{\alpha_{D}-\alpha_{U}}{\alpha_{A}-\alpha_{U}}+m
$$

yielding

$$
\begin{aligned}
\alpha_{f} & =\ell\left(\alpha_{D}-\alpha_{U}\right)+m\left(\alpha_{A}-\alpha_{U}\right)+\alpha_{U} \\
& =\ell \alpha_{D}+m \alpha_{A}+(1-\ell-m) \alpha_{U}
\end{aligned}
$$

The convective term using the nodal values is

$$
\begin{aligned}
C_{f} \alpha_{f}= & \max \left(0, C_{f}\right)\left[\ell_{f} \alpha_{P}+m_{f} \alpha_{F}+\left(1-\ell_{f}-m_{f}\right) \alpha_{U}^{+}\right]- \\
& \max \left(-C_{f}, 0\right)\left[\ell_{f} \alpha_{F}+m_{f} \alpha_{C}+\left(1-\ell_{f}-m_{f}\right) \alpha_{U}^{-}\right]
\end{aligned}
$$

The last expression allows the full implicit treatment of the HR schemes, so the values of the far nodes $\alpha_{U}^{+}$and $\alpha_{U}^{-}$ are actual nodes in the computational domain that can be resolved in the algebraic equation. For the one-dimensional structured grid, as shown in Fig. 2, the NWF form of the algebraic equation becomes

$$
\left(A_{P(\text { time })}+A_{P}\right) \alpha_{P}=\sum_{F \sim E, W, E E, W W} A_{F} \alpha_{F}+b_{P}
$$

where

$$
\begin{aligned}
& A_{E}=-\left[\begin{array}{l}
\max \left(0, C_{e}\right) m_{e}-\max \left(-C_{e}, 0\right) \ell_{e}+ \\
\max \left(0, C_{w}\right)\left(1-\ell_{w}-m_{w}\right)
\end{array}\right] \\
& A_{W}=-\left[\begin{array}{l}
\max \left(0, C_{w}\right) m_{w}-\max \left(-C_{w}, 0\right) \ell_{w}+ \\
\max \left(0, C_{e}\right)\left(1-\ell_{e}-m_{e}\right)
\end{array}\right] \\
& A_{E E}=\max \left(-C_{e}, 0\right)\left(1-\ell_{e}-m_{e}\right) \\
& A_{W W}=\max \left(-C_{w}, 0\right)\left(1-\ell_{w}-m_{w}\right) \\
& A_{P}=A_{E}+A_{W}+A_{E E}+A_{W W}+\left(C_{e}+C_{w}\right)
\end{aligned}
$$

$A_{P(\text { time })}$ and $b_{P}$ remain as defined in the last part of Section 3.

The above coefficients form a diagonally dominant matrix in which $A_{P}$ is always greater than zero because for almost all HR schemes, $\ell$ is greater than $m$. Only for the DD scheme $[\ell, m]=[0,1], A_{P}$ becomes zero. In this case, $[\ell, m]$ is set to $\left[L, 1-L \alpha_{f}\right]$ where $L$ is the value of $\ell$ from the previous interval of the composite scheme.

The NWF formulation is more robust than the DWF and faster than the DC method (Moukalled et al., 2016; Chourushi, 2018). However, the discretization above involves a pentagonal stencil that includes the far nodes in each coordinate direction which result in a pentadiagonal coefficient matrix.

\subsection{Reviewed Normalized Weighting Factor (RNWF) method}

The Reviewed Normalized Weighting Factor method developed by Chourushi (2018) is a tridiagonal version of the previously described NWF method. From the discrete equation (18), the change from pentadiagonal to tridiagonal stencil was performed considering the far nodes $E E$ and $W W$ as explicit. Then, these nodal values with their $A_{E E}$ and $A_{W W}$ coefficients are placed in the source term. Also, $A_{E E}$ and $A_{W W}$ are removed from the $A_{P}$ coefficient and added to the source term, resulting 
in the following modification of the previous $A_{P}$ and $b_{P}$ terms:

$$
\begin{aligned}
& A_{P}=A_{E}+A_{W}+\left(C_{e}+C_{w}\right) \\
& b_{P}=b_{P(\text { time terms })}+A_{E E}\left(\alpha_{E E}^{n}-\alpha_{P}^{n}\right)+A_{W W}\left(\alpha_{W W}^{n}-\alpha_{P}^{n}\right)
\end{aligned}
$$

In addition, to improve the numerical stability, the $[\ell, m]$ factors in the case of the DD scheme are changed to $\left[L, 1-L \tilde{\alpha}_{D}\right]$.

The RNWF method was 4 times faster than DC and 1.3 times faster than NWF for the test cases studied in Chourushi (2018). However, for our case, the numerical implementation of the interface-capture schemes in the context of multiphase flows, the RNWF did not show the same excellent efficiency than in the cases studied by Chourushi and sometimes slightly altered the interface geometry.

\section{Modified Normalized Weighting Factor (MNWF) method}

Encouraged by the numerical stability and efficiency of the NWF method, we decided to review again its formulation to be used in the context of multiphase flows. Unlike the RNWF, the formulation of our new alternative called Modified Normalized Weighting Factor (MNWF) method starts from the initial formulation of the NWF method and not from its final discretized equation as the RNWF method does.

First, the values of $\ell$ and $m$ for the interface-capture scheme are determined according to Eq. (14) and following the blending concept of Eq. (7). The values of $\ell$ and $m$ for some popular blended HR schemes used in the context of multiphase flows are listed in Table 1.

Second, to ensure numerical consistency, the $m$ factor for the blended HR scheme is corrected with the CBC condition. For which $\tilde{\alpha}_{f(\text { blended HR) }}$ is explicitly calculated using the blended HR scheme, and then bounded as

$$
\min \left(\tilde{\alpha}_{D}, 1\right) \leqslant \tilde{\alpha}_{f(\text { blended HR })} \leqslant \max \left(\tilde{\alpha}_{D}, 1\right)
$$

This bounded value is used to corrected $\mathrm{m}$ according to

$$
m_{\text {blended HR }}=\tilde{\alpha}_{f(\text { blended HR })}-\ell_{\text {blended HR }} \tilde{\alpha}_{D}
$$

Third, when $[\ell, m]_{\text {blended } \mathrm{HR}}=[0,1]$, the strategy introduced in the RNWF methodology is used instead of the original NWF strategy as it proved to be more numerically stable. Thus, the $[\ell, m]_{\text {blended } \mathrm{HR}}$ factors are set to $\left[L, 1-L \tilde{\alpha}_{D}\right]$ where $L$ is the $\ell$ factor of an interval of the diffusive HR scheme to preserve stability.

Fourth, following Eq. (15), Eq. (16) is obtained which is rewritten here for convenience as

$$
\alpha_{f}=\underbrace{\ell \alpha_{D}+m \alpha_{A}}_{\rightarrow A \text { coefficients }}+\underbrace{(1-\ell-m) \alpha_{U}}_{\text {Source term } s_{f}}
$$

Table $1 \quad \ell$ and $m$ factors in the NVD framework for some blended HR schemes used to advent the volume fraction

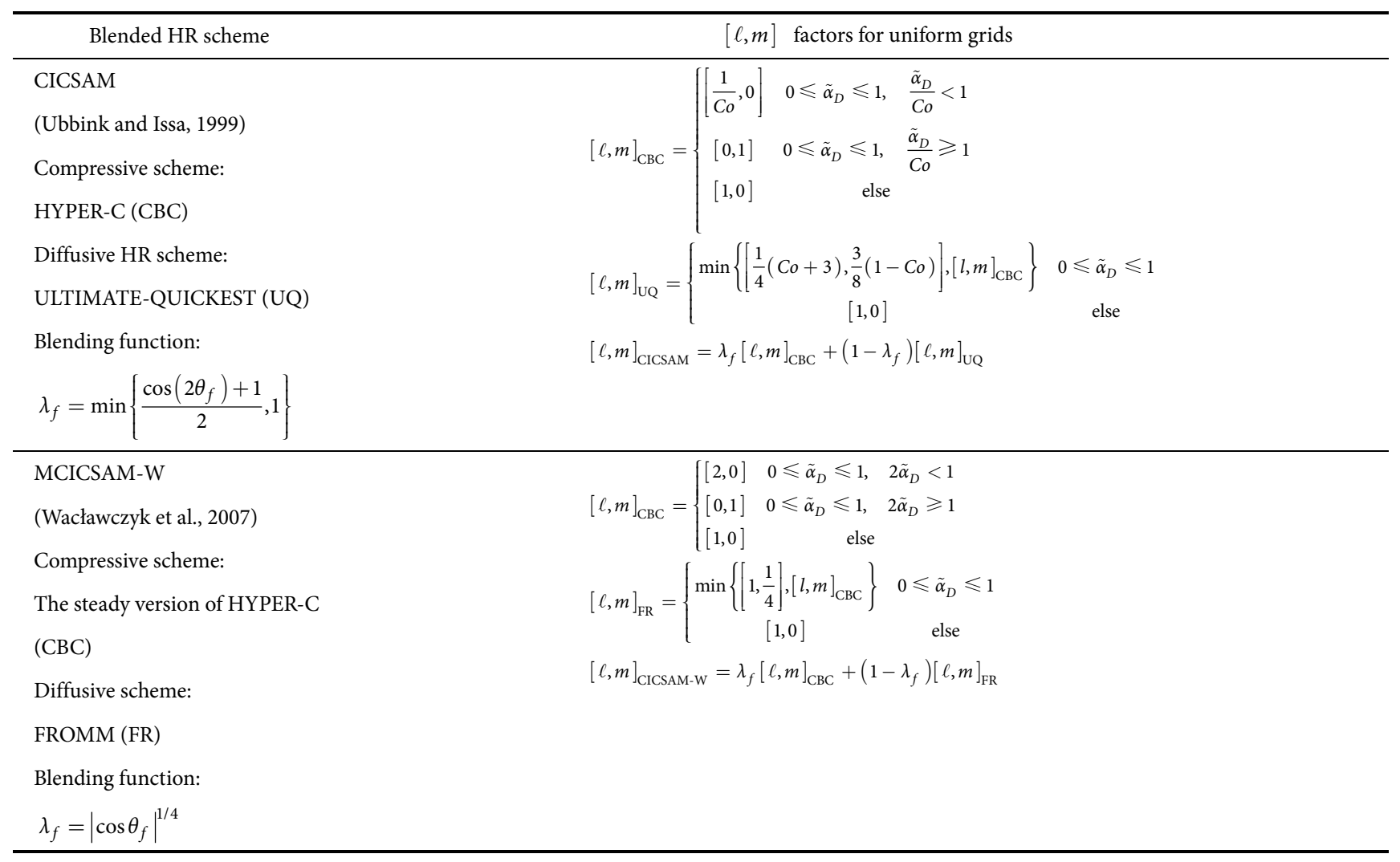


(Continued)

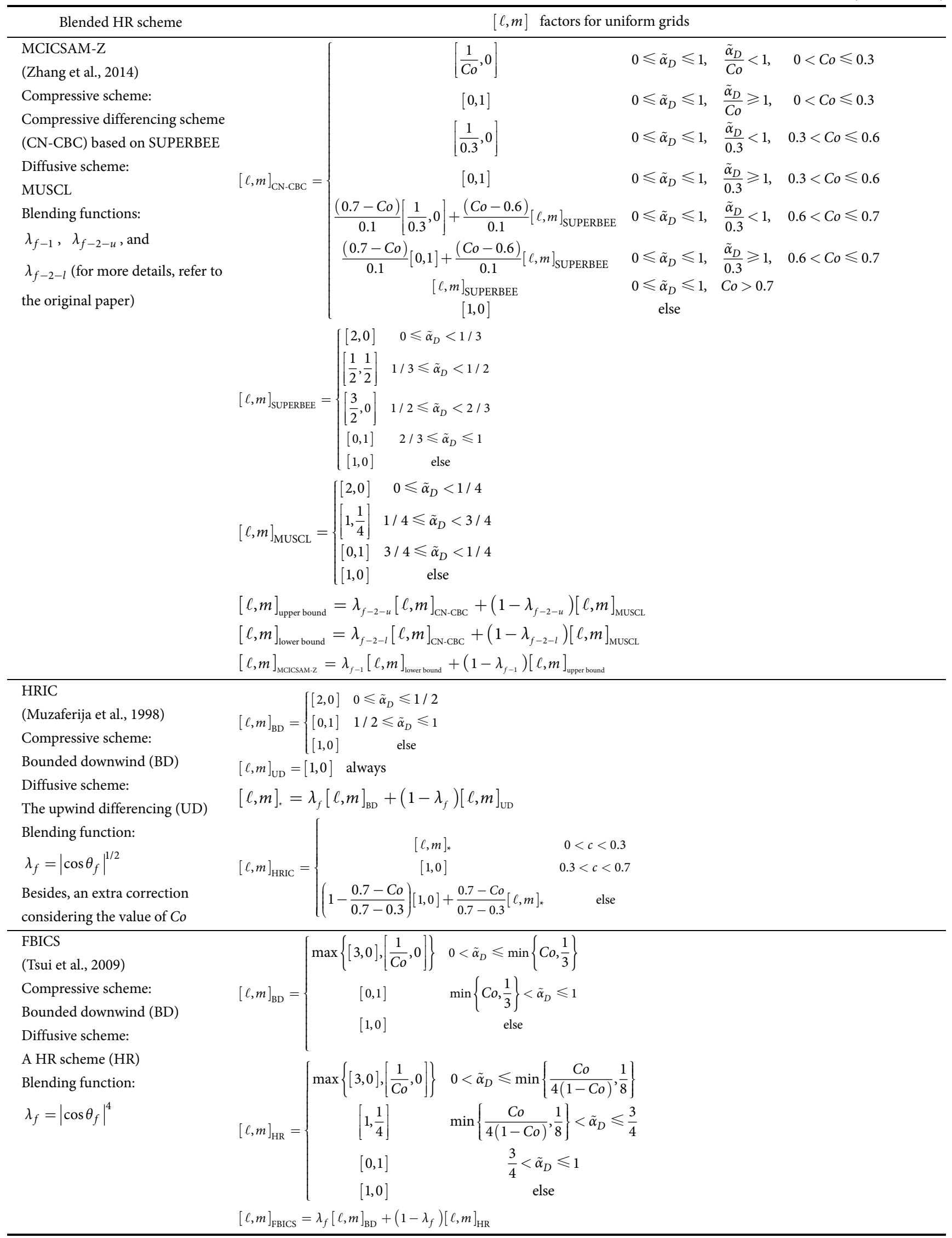


(Continued)

\begin{tabular}{|c|c|c|}
\hline Blended HR scheme & & {$[\ell, m]$ factors for uniform grids } \\
\hline $\begin{array}{l}\text { CUIBS } \\
\text { (Patel and Natarajan, 2015) } \\
\text { Compressive scheme: } \\
\text { Bounded downwind (BD) }\end{array}$ & {$[\ell, m]_{\mathrm{BD}}=$} & $\left\{\begin{array}{lc}{[3,0]} & 0<\tilde{\alpha}_{D} \leqslant \frac{1}{3} \\
{[0,1]} & \frac{1}{3}<\tilde{\alpha}_{D} \leqslant 1 \\
{[1,0]} & \text { else }\end{array}\right.$ \\
\hline $\begin{array}{l}\text { Diffusive scheme: } \\
\text { A HR scheme (HR) } \\
\text { Blending function: } \\
\lambda_{f}=\left|\cos \theta_{f}\right|^{1 / 4}\end{array}$ & {$[\ell, m]_{\mathrm{HR}}=$} & $\begin{cases}{[3,0]} & 0<\tilde{\alpha}_{D} \leqslant \frac{2}{13} \\
{\left[\frac{5}{6}, \frac{1}{3}\right]} & \frac{2}{13}<\tilde{\alpha}_{D} \leqslant \frac{4}{5} \\
{[0,1]} & \frac{4}{5}<\tilde{\alpha}_{D} \leqslant 1 \\
{[1,0]} & \text { else }\end{cases}$ \\
\hline & {$[\ell, m]_{\mathrm{CUIBS}}$} & $=\lambda_{f}[\ell, m]_{\mathrm{BD}}+\left(1-\lambda_{f}\right)[\ell, m]_{\mathrm{HR}}$ \\
\hline
\end{tabular}

At this point of the NWF formulation, we decided to use only the terms related to the donor and acceptor nodal values to form the matrix coefficients of $A$, while the last term involving the far node $U$ is explicitly determined and added directly to the source term as is shown in Moukalled et al. (2016). Hence, the expression in terms of nodal values Eq. (17) is used to obtain the following algebraic equation:

$$
\left(A_{P(\text { time })}+A_{P}\right) \alpha_{P}=\sum_{F \sim N B(P)} A_{F} \alpha_{F}+b_{P}
$$

where

$$
\begin{aligned}
A_{F} & =-\left[\max \left(0, C_{f}\right) m_{f}-\max \left(-C_{f}, 0\right) \ell_{f}\right] \\
A_{P} & =\sum_{f \sim n b(P)}\left[\max \left(0, C_{f}\right) \ell_{f}-\max \left(-C_{f}, 0\right) m_{f}\right] \\
S_{f} & =\left(1-\ell_{f}-m_{f}\right)\left[\max \left(0, C_{f}\right) \alpha_{U}^{+(n)}-\max \left(-C_{f}, 0\right) \alpha_{U}^{-(n)}\right] \\
b_{P} & =b_{P(\text { time })}-\sum_{f \sim n b(P)} S_{f}
\end{aligned}
$$

Finally, for a better explanation of the MNWF method, we give the algebraic equation for the one-dimensional structured grid presented in Fig. 2:

$$
\left(A_{P(\text { time })}+A_{P}\right) \alpha_{P}=\sum_{F \sim E, W} A_{F} \alpha_{F}+b_{P}
$$

where

$$
\begin{aligned}
A_{E}= & -\left[\max \left(0, C_{e}\right) m_{e}-\max \left(-C_{e}, 0\right) \ell_{e}\right] \\
A_{W}= & -\left[\max \left(0, C_{w}\right) m_{w}-\max \left(-C_{w}, 0\right) \ell_{w}\right] \\
A_{P}= & A_{P(\text { time })}+\left[\max \left(0, C_{e}\right) \ell_{e}-\max \left(-C_{e}, 0\right) m_{e}\right]+ \\
& {\left[\max \left(0, C_{w}\right) \ell_{w}-\max \left(-C_{w}, 0\right) m_{w}\right] } \\
S_{e}= & \left(1-\ell_{e}-m_{e}\right)\left[\max \left(0, C_{e}\right) \alpha_{W}^{n}-\max \left(-C_{e}, 0\right) \alpha_{E E}^{n}\right] \\
S_{w}= & \left(1-\ell_{w}-m_{w}\right)\left[\max \left(0, C_{w}\right) \alpha_{W W}^{n}-\max \left(-C_{e}, 0\right) \alpha_{E}^{n}\right] \\
b_{P}= & b_{P(\text { time })}-\left(S_{e}+S_{w}\right)
\end{aligned}
$$

\section{Results and discussion}

In this section, four test cases are presented to demonstrate the accuracy and efficiency of the new Modified Normalized Weighting Factor (MNWF) method. The results are compared with the traditional techniques Deferred Corrector (DC), Downwind Weighting Factor (DWF), and the recent alternative the Reviewed Normalized Weighting Factor (RNWF) method. For this purpose, the six blended highresolution schemes described above (CICSAM, MCICSAMW, MCICSAM-Z, HRIC, FBICS, and CUIBS) were implemented employing the four techniques. The efficiency is related to the computational effort of each technique; in other words, the total number of iterations required to achieve the convergence criterion during the simulation. The solution is assumed to have converged when the normalized residual defined by Eq. (28) is less than the chosen convergence criterion $(\zeta)$ :

$$
R^{\alpha}=\frac{\max _{\text {all cells }}\left|A_{P} \alpha_{P}-\sum_{F \sim N B_{P}} A_{F} \alpha_{F}-b_{P}\right|}{\max _{\text {all cells }}\left|A_{P} \alpha_{P}\right|} \leqslant \zeta
$$

For all the cases, no under-relaxation factor is used, and the maximum number of iterations per time step is limited to 20, whereas the convergence criterion is different for each case.

The solution algorithm employed for all the cases is a sequential one. At each time-step, the volume fraction equation (3) is firstly solved at the beginning of each time-step. Then, the new volume fraction field is used to compute the local density and viscosity using Eq. (4), and finally, the momentum and continuity equations (1) and (2) are solved by the predictor-corrector SIMPLE algorithm.

The first two test cases are the advection of the slotted circle in a rotational flow field introduced by Zalesak (1979) and the advection of a circle in a shear flow presented by 
Rudman (1997) which have simple exact solutions. These are frequently used in the multiphase community to check the performance of the advection schemes dealing with a non-uniform distribution of the Courant number and a considerable interface deformation, respectively (Ubbink and Issa, 1999; Zhang et al., 2014; Patel and Natarajan, 2015). For both, the accuracy of the simulation results is verified using the root mean square (RMS) error defined as

$$
\text { error }=\sqrt{\frac{\sum_{i}^{N}\left(\alpha_{i}^{n}-\alpha_{i}^{a}\right)^{2}}{N}}
$$

where $\alpha_{i}^{n}$ is the numerical solution, $\alpha_{i}^{a}$ is the exact solution, and $N$ is the total number of control volumes. The initialization of the volume fraction field for these cases are illustrated in Fig. 3.

\subsection{Advection of a Slotted Circle in a rotational flow}

A circle with a diameter of $1 \mathrm{~m}$ and a slot of width $0.12 \mathrm{~m}$ and depth $0.62 \mathrm{~m}$ is centered at $(2,2.65) \mathrm{m}$ of a square $4 \mathrm{~m} \times$ $4 \mathrm{~m}$ domain, and exposed to a clockwise circular velocity given by

$$
u=-\omega\left(y-y_{0}\right), \quad v=\omega\left(x-x_{0}\right)
$$

where $\left(x_{0}, y_{0}\right)=(2,2)$ is the center of the rotation and $\omega=$ $0.5 \mathrm{rad} / \mathrm{s}$ is the constant angular velocity. The time needed for one rotation is $12.57 \mathrm{~s}$. The problem is discretized with a structured grid of $200 \times 200$ square control volumes, and it is solved for five different time-step sizes which produce a maximum Courant number $(\mathrm{Co})$ of $0.2,0.4,0.6$, and 0.8 at point $(2,2.15) \mathrm{m}$ (Darwish and Moukalled, 2006). The convergence criterion is $\zeta=5 \times 10^{-3}$.

The stacked columns shown in Fig. 4 represent the total number of iterations that each HR scheme performed to converge during one rotation of the slotted circle at different maximum Courant numbers. The bottom layer of the column represents the number of iterations performed using the DC method, the second relates to the DWF, the third to RNWF,

(a)

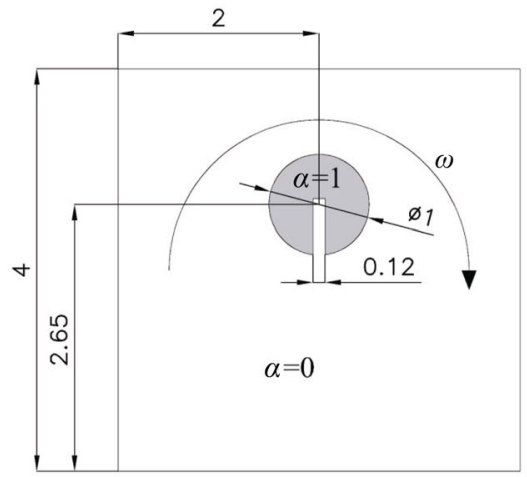

and the last to MNWF. The results for the six different HR schemes are displayed in (a) to (f), with CICSAM shown in (a), MCICSAM-W in (b), MCICSAM-Z in (c), HRIC in (d), FBICS in (e), and CUIBS in (f). The efficiency between the four methods varies according to the HR scheme. However, for all of them, the new MNWF is the fastest to reach the convergence, whereas the DWF method is the slowest. For example, for CICSAM and MCICSAM-Z, the MNWF is about 20\%-35\% faster than DC and RNWF, and 35\%-80\% faster than DWF. Whereas for the HRIC and MCICSAM-W scheme, the MNWF works similarly to DC and RNWF but is noticeably better than DWF with about 20\%-85\% fewer total iterations. In the cases of FBICS and CUIBS, the total number of iterations used by MNWF is from $12 \%-25 \%$ less than by DC and RNWF, while 10\%-85\% less than for DWF.

On the one hand, for low Co, the influence of the implementation method is negligible for all schemes. On the other hand, for medium and high Co, the implementation method plays a significant role. For instance, the MNWF is about 10\%-30\% more efficient than DC and RNWF, and $50 \%-85 \%$ than DWF.

Table 2 shows the accuracy of each HR scheme implemented through the four different techniques. For CICSAM, HRIC, and MCICSAM-W, the error varies according to the implementation technic used, and it is more significant for high Co. Nonetheless, the best performance remains in MNWF. To visualize this difference in accuracy, Fig. 5 shows the contour plots at $C o=0.8$ of these three schemes. For the other three HR schemes, the error is independent of the implementation method.

\subsection{Advection of a circle in a shear flow}

The volume fraction field is initialized, as is shown in Fig. $3(\mathrm{~b})$. A circle of $0.2 \pi \mathrm{m}$ diameter with its center at $(0.5 \pi$, $0.2(1+\pi)) \mathrm{m}$ filled with phase one is in a square domain of phase two. The two-phase configuration is exposed to a shear flow field described by

(b)

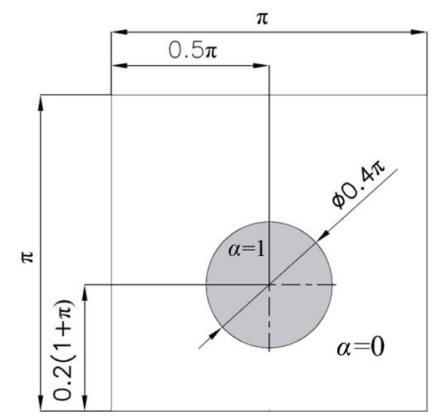

Fig. 3 Initial configuration for (a) the slotted circle test case and (b) the circle in a shear flow. 
(a) CICSAM

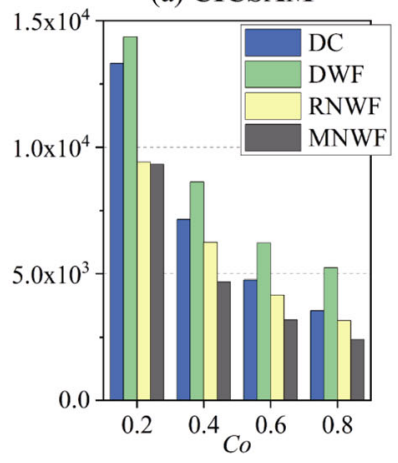

(d) HRIC

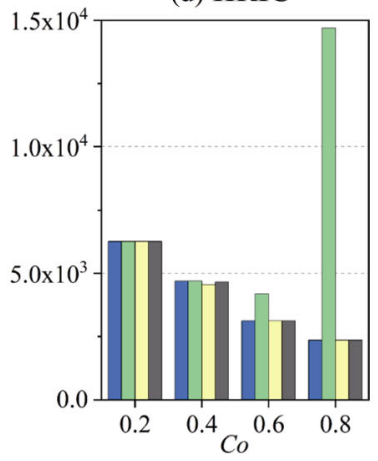

(b) MCICSAM-W

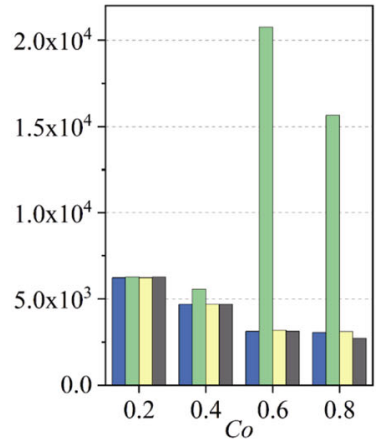

(e) FBICS

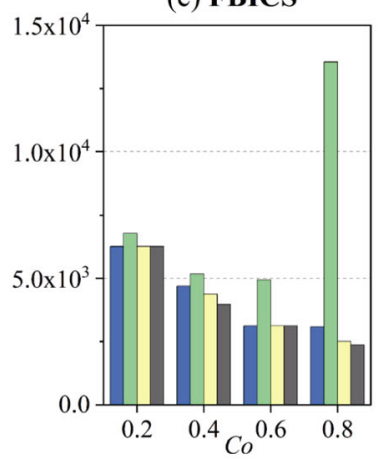

(c) MCICSAM-Z

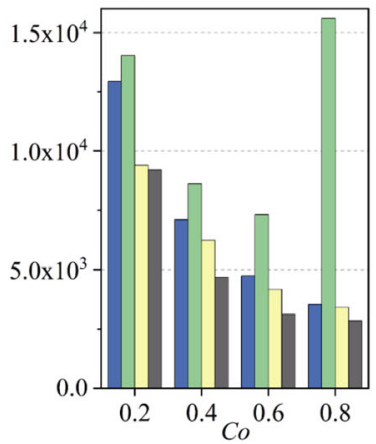

(f) CUIBS

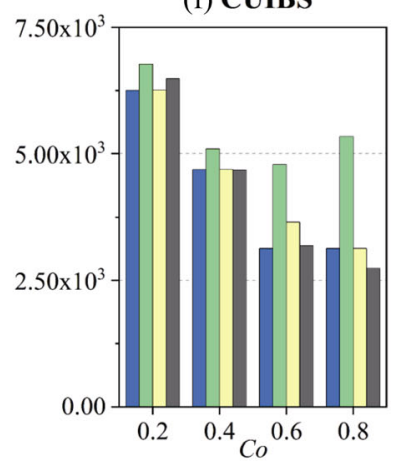

Fig. 4 Comparison of the total number of iterations required for the convergence of (a) CICSAM, (b) HRIC, (c) MHRIC, (d) MCICSAM, (e) FBICS, and (f) CUIBS implemented using the DC, DWF, RNWF, and the new MNWF methods at different maximum Courant numbers.

Table 2 RMS error after one rotation of the slotted circle

\begin{tabular}{|c|c|c|c|c|c|c|c|c|c|c|c|c|}
\hline \multirow{2}{*}{$\frac{\text { HR scheme }}{\text { Co }}$} & \multicolumn{4}{|c|}{$\operatorname{CICSAM}\left(10^{-2}\right)$} & \multicolumn{4}{|c|}{ MCICSAM-W $\left(10^{-2}\right)$} & \multicolumn{4}{|c|}{ MCICSAM-Z $\left(10^{-2}\right)$} \\
\hline & DC & DWF & RNWF & MNWF & $\mathrm{DC}$ & DWF & RNWF & MNWF & DC & DWF & RNWF & MNWF \\
\hline 0.2 & 1.55 & 1.63 & 15.50 & 1.54 & 2.64 & 2.41 & 2.54 & 2.78 & 1.21 & 1.21 & 1.21 & 1.21 \\
\hline 0.4 & 1.69 & 1.87 & 7.53 & 1.48 & 2.62 & 2.42 & 2.51 & 2.40 & 1.28 & 1.28 & 1.28 & 1.28 \\
\hline 0.6 & 2.09 & 2.23 & 6.08 & 1.82 & 2.93 & 2.35 & 2.59 & 2.80 & 1.46 & 1.46 & 1.46 & 1.46 \\
\hline 0.8 & 3.36 & 3.38 & 5.35 & 3.25 & 3.00 & 2.88 & 2.90 & 2.44 & 2.10 & 2.10 & 2.10 & 2.10 \\
\hline HR scheme & \multicolumn{4}{|c|}{ HRIC $\left(10^{-2}\right)$} & \multicolumn{4}{|c|}{$\operatorname{FBICS}\left(10^{-2}\right)$} & \multicolumn{4}{|c|}{ CUIBS $\left(10^{-2}\right)$} \\
\hline Co & DC & DWF & RNWF & MNWF & $\mathrm{DC}$ & DWF & RNWF & MNWF & DC & DWF & RNWF & MNWF \\
\hline 0.2 & 2.87 & 2.79 & 2.78 & 2.79 & 1.65 & 1.65 & 1.65 & 1.65 & 1.58 & 1.58 & 1.58 & 1.58 \\
\hline 0.4 & 2.85 & 2.79 & 2.70 & 2.79 & 1.67 & 1.67 & 1.67 & 1.67 & 1.60 & 1.60 & 1.60 & 1.60 \\
\hline 0.6 & 2.88 & 2.71 & 2.74 & 2.71 & 1.85 & 1.85 & 1.85 & 1.85 & 1.67 & 1.67 & 1.67 & 1.67 \\
\hline 0.8 & 3.26 & 2.81 & 3.09 & 2.79 & 1.60 & 1.60 & 1.60 & 1.60 & 1.64 & 1.64 & 1.64 & 1.64 \\
\hline
\end{tabular}

$u=\sin (x) \cos (y), \quad v=-\cos (x) \sin (y)$

where $x, y \in[0, \pi]$. The domain discretized with a uniform structured mesh consisting of $160 \times 160$ cells, and the time- step is chosen so that the local Courant number is 0.5 . For observing the performance of the HR schemes together with the implementation method in the presence of interface deformation, the simulation is firstly run for $n$ time-steps using the velocity defined in Eq. (31), then the flow is reversed, and the simulation is rerun for $n$ time- steps. Hence, the interface should return to its initial shape. For this study, $n=1000$ and $n=2000$ are investigated.

Figure 6 summarizes the total number of iterations required by the $\mathrm{HR}$ schemes implemented with the four methods DC, DWF, RNWF, and MNWF in the case of (a) $n=1000$ and (b) $n=2000$. The convergence criterium for these simulations is $\zeta=5 \times 10^{-3}$. In case (a), 1000 forward steps followed by 1000 backward steps, the MNWF is $15 \%-50 \%$ faster than DC, $45 \%-84 \%$ faster than DWF, and 
CICSAM
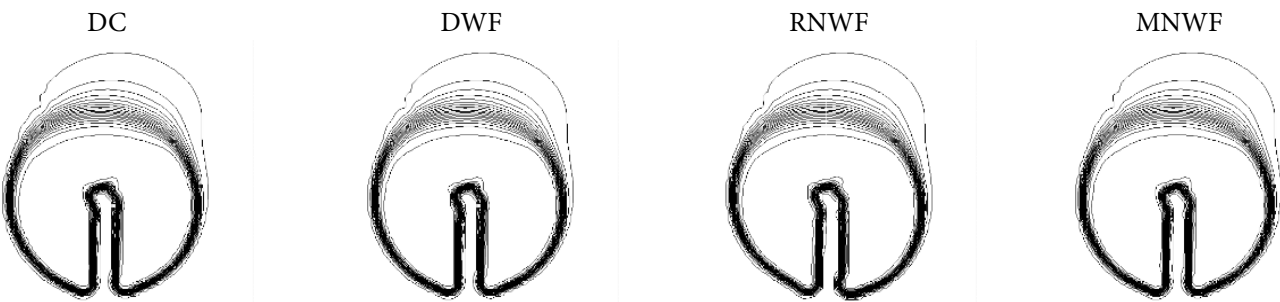

MCICSAM-W
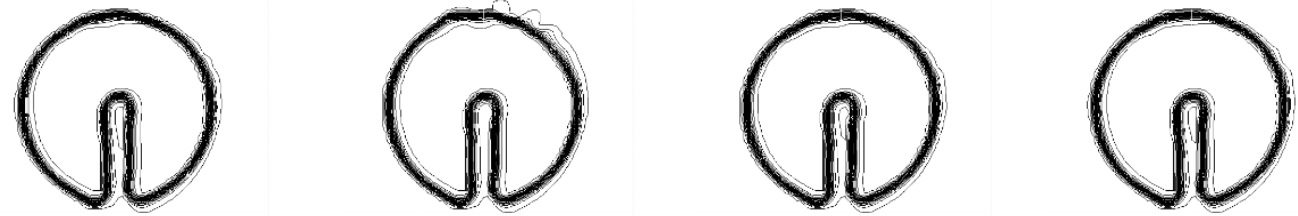

HRIC
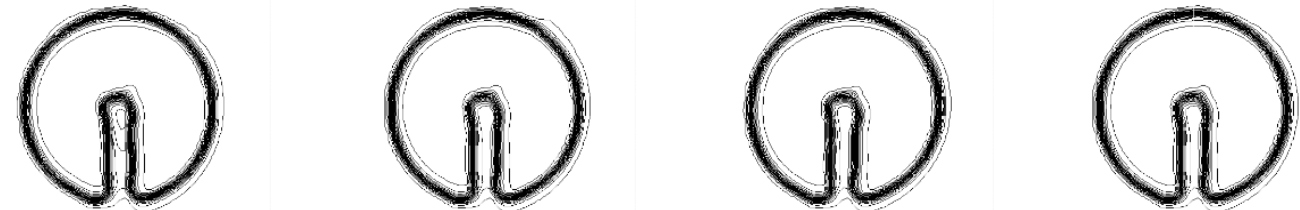

Fig. 5 Contour plots of the slotted circle problem after one complete rotation at maximum Co $=0.8$ for three blended HR schemes.

(a)

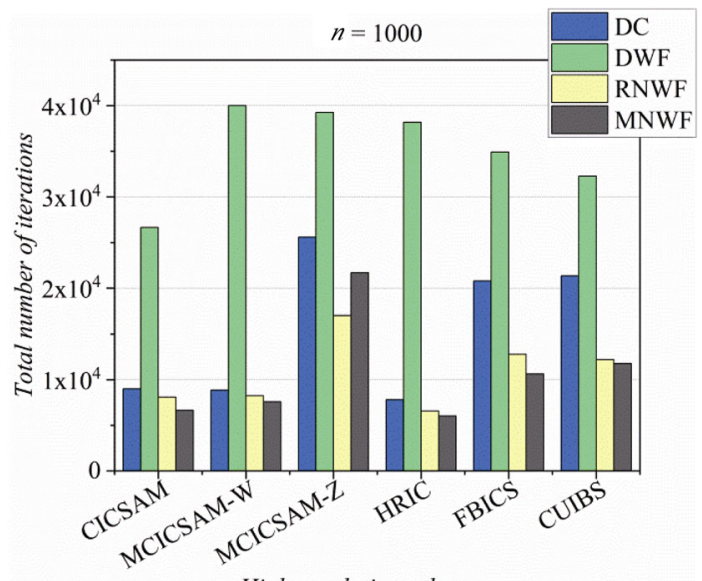

(b)

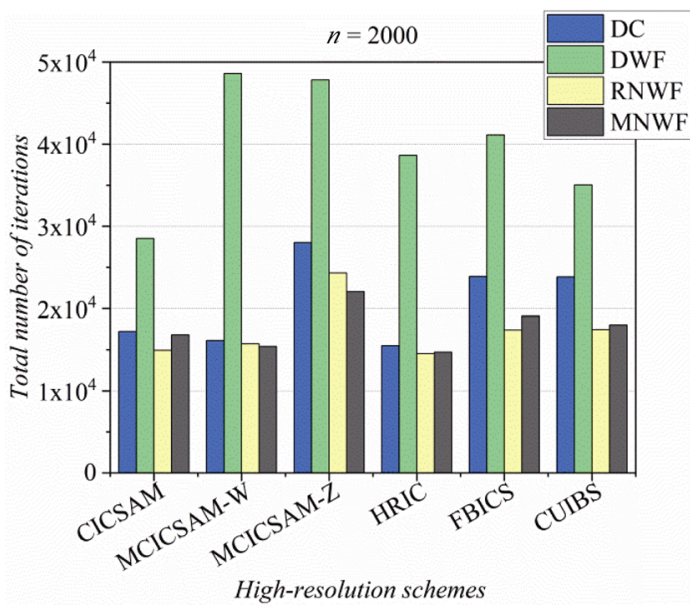

Fig. 6 Total number of iterations for the circle in a shear flow test case using the different HR schemes implemented employing the DC, DWF, RNWF, and MNWF methods. (a) After 1000 forward steps, followed by 1000 backward steps. (b) After 2000 forward steps, followed by 2000 backward steps.

about 3\%-18\% faster than RNWF. Only for MCICSAM-Z, the RNWF seems to be a better alternative. In case (b), 2000 steps forward followed by 2000 steps backwards, the high degree of interface deformation reduces the efficiency of the MNWF. Thus, the computational effort between MNWF and RNWF are similar, and the MNWF is now only $5 \%-27 \%$ faster than DC and $45 \%-58 \%$ faster than DWF. Nevertheless, MNWF is still a good option for the implementation of the analyzed schemes.

Table 3 contains the errors of these simulations, which are slightly different for all schemes and implementation techniques. For $n=1000$, the six advection schemes almost recover the initial shape. Therefore, the errors are small and fluctuate between $1.83 \times 10^{-2}$ and $4.55 \times 10^{-2}$. While for $n=$ 2000 , all schemes suffer from numerical diffusion, independently of the implementation technique. Thus, the errors are more significant and range from $8.87 \times 10^{-2}$ to $11.4 \times 10^{-2}$. Figure 7 depicts the results of two of the tested schemes: CUIBS (low error values) and CICSAM (high error values) for (a) $n=$ 1000, and (b) $n=2000$ forward and backward steps.

\subsection{Rising bubble}

The third test case, the rising bubble, is one of the multiphase flow benchmark cases of Hysing et al. (2009) which is an example of bubble dynamics with strong surface tension effects. Figure 8 illustrates the initial configuration 
Table 3 RMS error for $n$ forward and $n$ backward steps of the circle in shear flow test case

\begin{tabular}{|c|c|c|c|c|c|c|c|c|}
\hline \multirow{2}{*}{ HR scheme } & \multicolumn{4}{|c|}{$\begin{array}{l}\text { After } 1000 \text { steps forward followed by } 1000 \text { steps } \\
\text { backwards }\left(10^{-2}\right)\end{array}$} & \multicolumn{4}{|c|}{$\begin{array}{l}\text { After } 2000 \text { steps forward followed by } 2000 \text { steps } \\
\text { backwards }\left(10^{-2}\right)\end{array}$} \\
\hline & $\mathrm{DC}$ & DWF & RNWF & MNWF & $\mathrm{DC}$ & DWF & RNWF & MNWF \\
\hline CICSAM & 4.51 & 4.53 & 4.55 & 4.55 & 9.52 & 9.43 & 10.80 & 9.46 \\
\hline MCICSAM-W & 3.34 & 3.26 & 3.31 & 3.34 & 9.47 & 9.00 & 10.20 & 9.17 \\
\hline MCICSAM-Z & 1.91 & 1.90 & 2.28 & 3.73 & 8.98 & 8.92 & 10.60 & 9.10 \\
\hline HRIC & 3.71 & 3.62 & 3.61 & 3.65 & 9.29 & 9.15 & 10.10 & 9.12 \\
\hline FBICS & 1.83 & 1.90 & 2.09 & 2.15 & 8.88 & 8.87 & 8.93 & 10.20 \\
\hline CUIBS & 1.85 & 1.93 & 1.91 & 1.89 & 8.90 & 8.89 & 11.40 & 8.89 \\
\hline
\end{tabular}

(a)

CICSAM

CUIBS

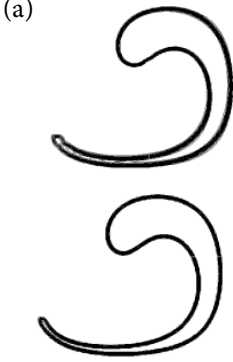

(b)

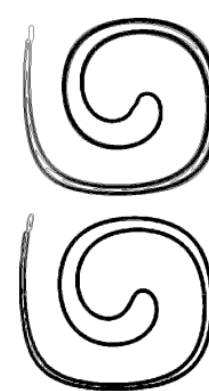

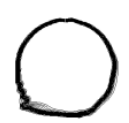

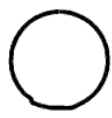

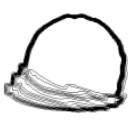

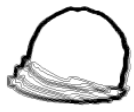

Fig. 7 Results of the circle in a shear flow test case for the CICSAM and CUIBS scheme implemented using MWFM. (a) After 1000 forward steps, followed by 1000 backward steps. (b) After 2000 forward steps, followed by 2000 backward steps.

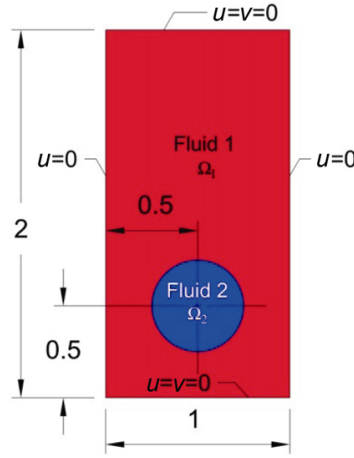

Fig. 8 Initial configuration and boundary conditions for the rising bubble problem.

and boundary conditions of the problem.

A $0.5 \mathrm{~m}$ diameter bubble of fluid 2 is positioned at $(0.5$, $0.5) \mathrm{m}$ within a tank of $1 \mathrm{~m} \times 2 \mathrm{~m}$ filled with fluid 2 . The top and bottom walls are defined as no-slip boundary conditions and the vertical walls as symmetry boundary conditions. The density and dynamic viscosity of fluid 1 are $\rho_{1}=1000 \mathrm{~kg} / \mathrm{m}^{3}$ and $\mu_{1}=10 \mathrm{~kg} /(\mathrm{m} \cdot \mathrm{s})$, and for fluid $2 \rho_{2}=$ $100 \mathrm{~kg} / \mathrm{m}^{3}$ and $\mu_{2}=1 \mathrm{~kg} /(\mathrm{m} \cdot \mathrm{s})$. The dynamic of the bubble is controlled by a gravity force $g=-0.98 \mathrm{~m} / \mathrm{s}^{2}$ and a surface tension coefficient $\sigma=24.5 \mathrm{~N} / \mathrm{m}$. The benchmark quantities are the temporal evolution during three seconds of the center of mass of the bubble and its mean rising velocity which are determined as

$$
y_{\mathrm{c}}=\frac{\sum_{i \in \Omega_{2}} y_{i} \delta V_{i}}{\sum_{i \in \Omega_{2}} \delta V_{i}}, \quad v_{\mathrm{c}}=\frac{\sum_{i \in \Omega_{2}} v_{i} \delta V_{i}}{\sum_{i \in \Omega_{2}} \delta V_{i}}
$$

where $\Omega_{2}$ denotes the region that the bubble occupies and $\delta V$ size of the control volume.

The above quantities are used to validate the multiphase code and also the quality of the overall solution method. The error of the simulation is quantified using the following relative error norm:

$$
\text { error }=\frac{\sum_{i=1}^{N}\left|q_{\mathrm{t}, \mathrm{ref}}-q_{\mathrm{t}}\right|}{\sum_{i=1}^{N}\left|q_{\mathrm{t}, \mathrm{ref}}\right|}
$$

where $q_{\mathrm{t}}$ is the temporal evolution of the quantity $x_{\mathrm{c}}$ or $y_{\mathrm{c}}$, and $q_{\text {ref }}$ is the reference solution presented in Hysing et al. (2009).

The computation is performed for three uniform structured grids which consist of $40 \times 80,80 \times 160$, and $160 \times 320$ hexahedral control volumes. The time steps selected for each mesh are $0.02 \mathrm{~s}, 0.01 \mathrm{~s}$, and $0.005 \mathrm{~s}$ respectably. This selection results in a maximum local Co of 0.45 for the three grids. The convergence criterion is $\zeta=5 \times 10^{-4}$ for the volume fraction field and $\zeta_{u}=10^{-6}$ for the velocity field. The curvature is calculated with the height-function method (Malik et al., 2007).

Figure 9 shows the total number of iterations for the coarse, medium, and fine grid during three seconds of the calculation for the six HR schemes implemented using the DC, DWF, RNWF, and MNWF methods. For the three girds in this test case, the efficiency of the DC, DWF, RNWF and MNWF methods are similar for CICSAM and MCICSAM-Z. Although for the coarse and medium grid, 
(a) Coarse grid $40 \times 80$

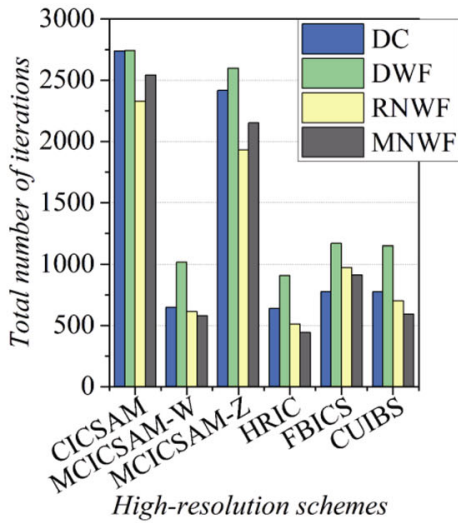

(b) Medium grid $80 \times 160$

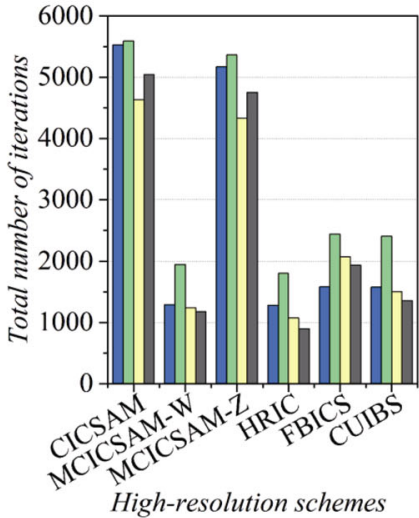

(c) Fine grid $160 \times 320$

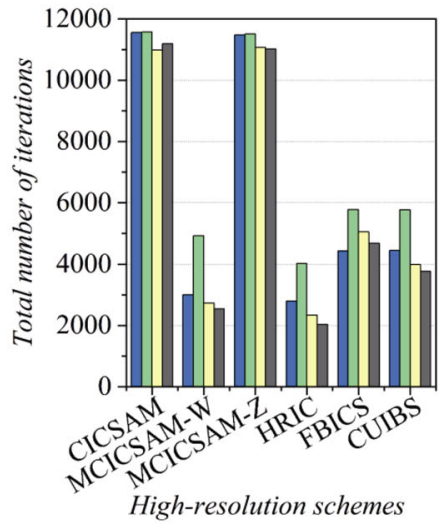

Fig. 9 Total number of iterations for (a) coarse grid, (b) medium grid, and (c) fine grid for the rising bubble test case. The six blended HR schemes implemented through the DC, DWF, RNWF, and MNWF methods are presented.

the RNWF seems to have a small advantage. On the other hand, for the three grids and the other HR schemes, the percentage difference between the four methods is similar. The MNWF method is $15 \%-27 \%$ faster than DC, $6 \%-13 \%$ than RNWF, and $20 \%-50 \%$ than DWF. Although the most influential part of this simulation is the calculation of the curvature, the effects of the implementation techniques remained, and again the MNWF shows the best performance and DWF the worst.

Besides that, the relative error of the results computed for the fine grid, presented in Table 4, reveals that the calculations performed using the MNWF method are in the same range of precision than with the other methods. Figure 10(a) shows the final position of the bubble solved

Table 4 Relative error norm for the rising bubble test case solved using the different HR schemes and the implementation techniques for the fine grid

\begin{tabular}{|c|c|c|c|c|c|c|c|c|}
\hline \multirow{2}{*}{ HR scheme } & \multicolumn{4}{|c|}{ Center of the mass $\left(10^{-3}\right)$} & \multicolumn{4}{|c|}{ Rising velocity $\left(10^{-3}\right)$} \\
\hline & $\mathrm{DC}$ & DWF & RNWF & MNWF & DC & DWF & RNWF & MNWF \\
\hline CICSAM & 4.67 & 4.69 & 4.69 & 4.69 & 3.52 & 3.52 & 3.50 & 3.42 \\
\hline MCICSAM-W & 3.67 & 3.70 & 3.69 & 3.67 & 11.00 & 11.00 & 10.90 & 11.00 \\
\hline MCICSAM-Z & 3.39 & 3.39 & 3.14 & 3.23 & 3.32 & 3.32 & 3.25 & 3.24 \\
\hline HRIC & 4.67 & 4.69 & 4.69 & 4.69 & 14.80 & 14.80 & 14.60 & 14.90 \\
\hline FBICS & 3.28 & 3.30 & 3.26 & 3.26 & 4.24 & 4.25 & 4.23 & 4.27 \\
\hline CUIBS & 3.27 & 3.29 & 3.30 & 3.28 & 4.08 & 4.08 & 4.04 & 4.08 \\
\hline
\end{tabular}

(a)

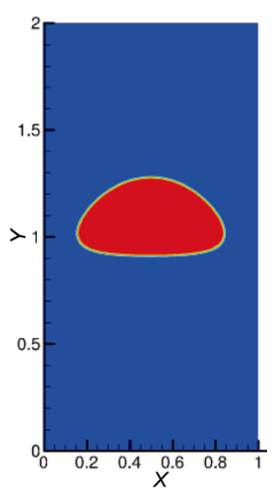

(b)

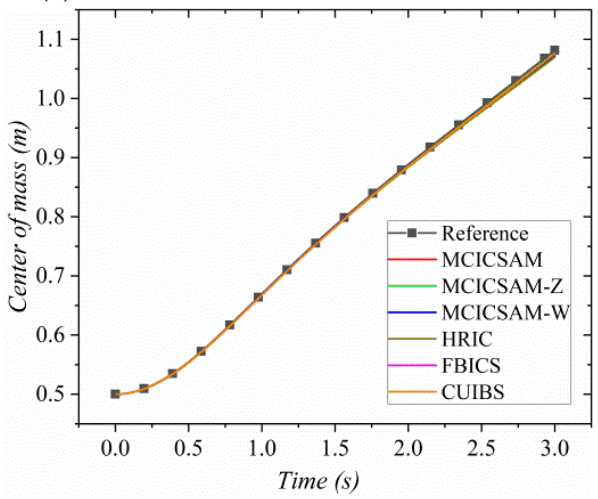

(c)

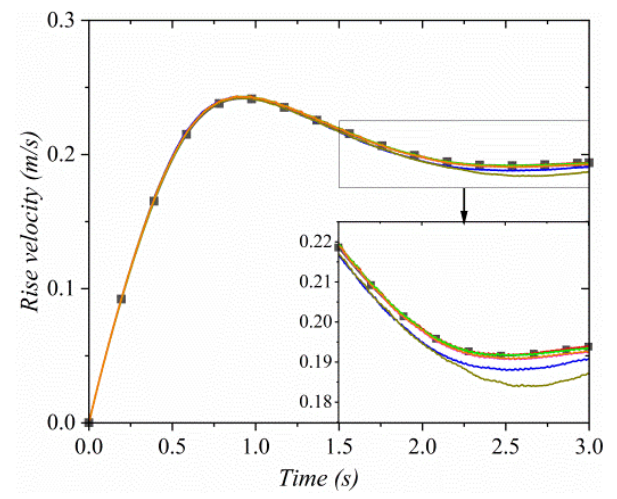

Fig. 10 (a) Final position of the bubble at $3 \mathrm{~s}$ calculated using the CUIBS scheme in combination with the MNWF method for the fine grid. (b) Center of mass and (c) rise velocity of the rising bubble during $3 \mathrm{~s}$ for the six blended HR schemes implemented employing the new MNWF method for the fine grid. The reference refers to the results of Hysing et al. (2009) 
with the CUIBS scheme in combination with MNWF for the fine grid, and (b) the evolution of the centre of mass with time, and (c) the rise velocity of the bubble for the six blended HR schemes implemented with the MNWF method.

\subsubsection{D rising bubble}

In order to measure the efficiency of the new approach in $3 \mathrm{D}$ scenarios, the $2 \mathrm{D}$ rising bubble test case was extended to $3 \mathrm{D}$ according to the configuration showed in Turek et al. (2019). The physical properties, gravity, and surface tension coefficient are the same as for the $2 \mathrm{D}$ case. Only now all walls are set as non-slip boundary conditions. The computational domain is a uniform structured grid of $80 \times 80 \times 160$ hexahedral control volumes divided into 32 blocks. The time step is $0.005 \mathrm{~s}$ which produces a maximum $C o \sim 0.55$. The curvature is calculated with the standard second-order central difference

(a)

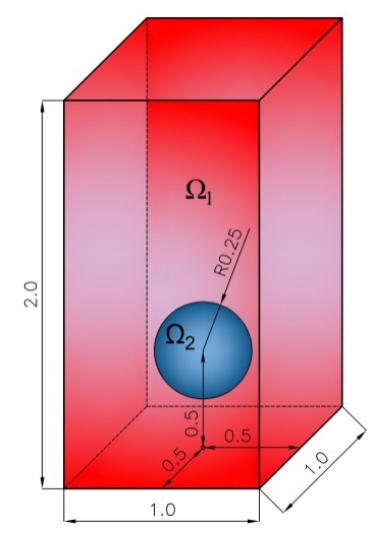

(b)

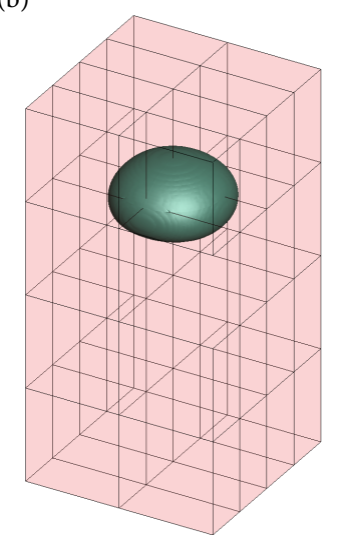

scheme (CDS). The convergence criterion is $\zeta=5 \times 10^{-4}$ for the volume fraction and $\zeta_{u}=10^{-7}$ for the velocity. For this part, the results obtained with CICSAM and MCICSAM-Z are not presented because their low performance shown in the $2 \mathrm{D}$ case is almost the same in the $3 \mathrm{D}$ case, and these impede the precise observation of the other results. Figure 11 shows the new initial geometric configuration, the simulation results of the position of the bubble at three seconds, and the evolution of the rise velocity. The four schemes MCICSAM-W, HRIC, FBICS, CUIBS, implemented with the MNWF method agree with the reference result published in Turek et al. (2019).

Concerning the efficiency of the MNWF method, the performance shown for the $2 \mathrm{D}$ case is almost the same for the $3 \mathrm{D}$ case. The MNWF method is the fastest and the DWF method the slowest. Except for the FBICS scheme where the quickest is the DC. See Fig. 12.

(c)

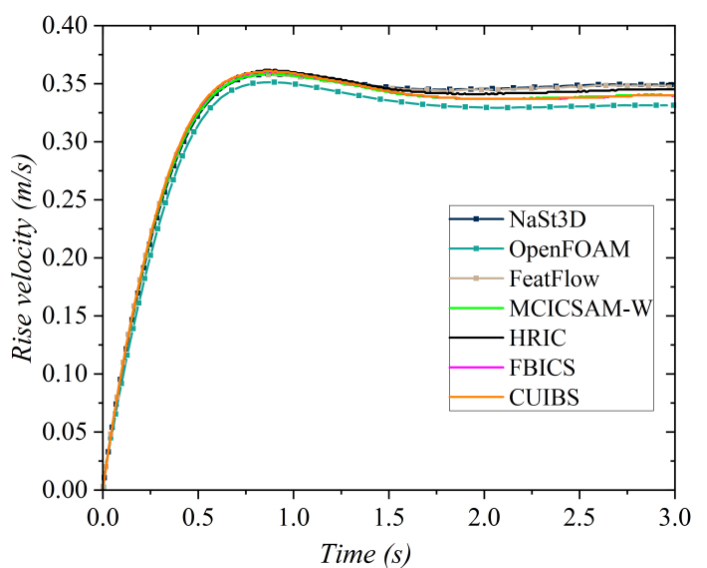

Fig. 11 (a) Geometric initial configuration for the 3D rising bubble test case, (b) bubble position at 3 s. The lines depict the division into blocks of the grid. (c) Temporal evolution of the rise velocity of the center of the bubble obtained with four methods implemented with MNWF and the reference results computed with NaSt3D, OpenFOAM, and FeatFlow.

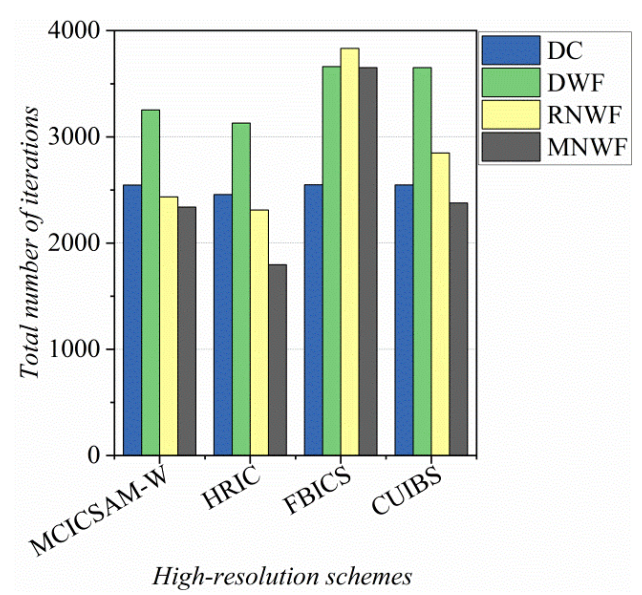

Fig. 12 Total number of iterations for the 3D rising bubble test case for the four blended HR schemes implemented using the DC, DWF, RNWF, and MNWF methods.

\subsection{Dam break flow impacting a rigid structure}

Finally, to validate our proposed MNWF method for more realistic applications, the classic dam breaking example is computed. It was experimentally studied by Koshizuka (1995) to describe the collapse of a water column impacting a rigid structure. Figure 13 represents the geometry and physical parameters of the problem. It consists of a box open to the atmosphere that contains a water column which collapses and hits a rigid obstacle. The high-density fluid is water, and the low-density fluid is air, both with standard physical properties. The two-phase flow is considered laminar, and the surface tension effects are neglected.

The computation is run for two grids consisting of hexahedral control volumes (CV), a coarse grid of $2384 \mathrm{CV}$ and a fine grid of $9536 \mathrm{CV}$. Each mesh is formed by five 


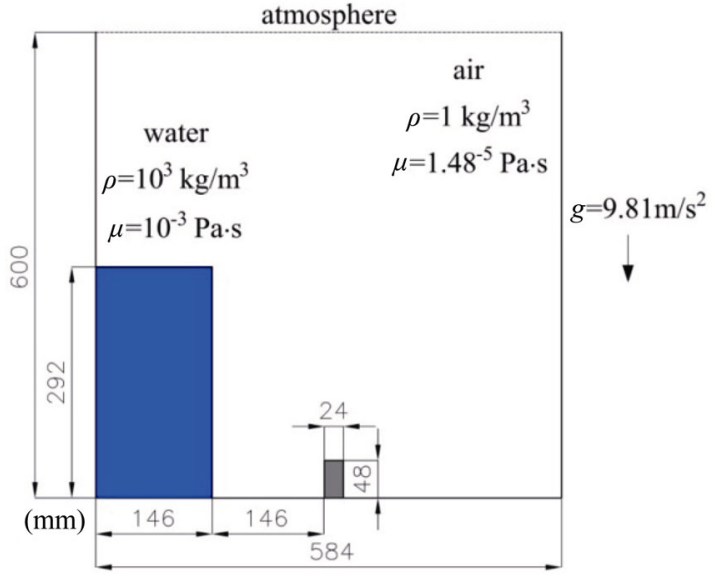

(a)

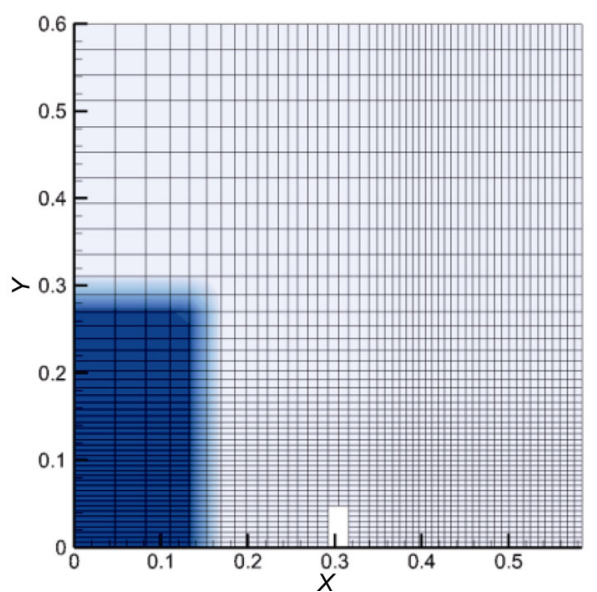

(b)

Fig. 13 (a) Geometrical configuration and physical parameters of the dam break flow impacting a rigid structure test case. The dimensions are in $\mathrm{mm}$. (b) Initial condition in the coarse computational domain.

blocks divided according to the contour of the obstacle and following a hyperbolic distribution as is shown in Fig. 13(b). The simulations are computed for $0.9 \mathrm{~s}$ with a variable time-step defined for each grid to maintain a $\mathrm{Co}$ below one. For these calculations, the adaptative time step is chosen because the kinematic energy of the water increases with the time, which produces a constant increase of the Courant number. If a small constant time step is selected, the calculation is stable but is inefficient, while a large value leads to divergence when the velocity of the water is higher. The convergence criterion for the volume fraction field is $\zeta=5 \times 10^{-4}$, and $\zeta_{u}=10^{-7}$ for the velocity and pressure.

Figure 14 presents the total number of iterations required in each grid for the six HR schemes implemented using DC, DWF, RNWF, and MNWF. For the two grids, the difference in the performance of the methods is less

(a) Coarse grid $(2384 \mathrm{CV})$

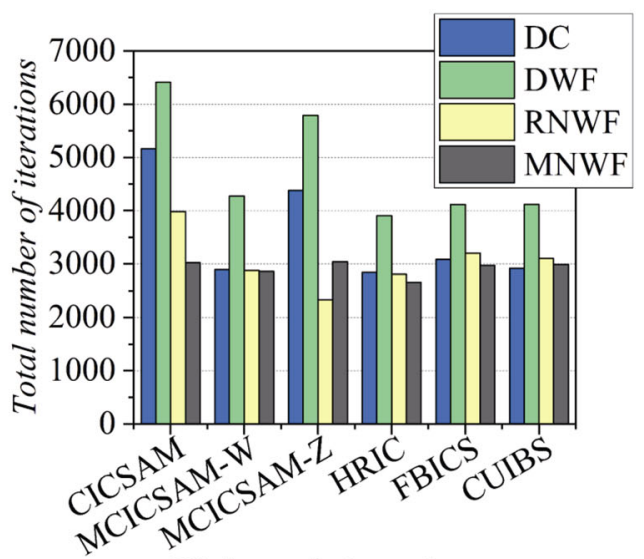

High-resolution schemes evident than for the other cases. It is because the timing control results in low Courant numbers over a large part of the simulation period, thus decreasing the difference in efficiency that exists between the methods. As shown in the first case, the advection of a Slotted Circle in a rotational flow, for low $\mathrm{Co}$, all four methods act similarly. However, for these grids, one observes that for the CICSAM and MCICSAM-Z schemes, the new MNWF method is considerably better than DC and DWF, and slightly better than RNWF. Although RNWF is the quickest for MCICSAM-Z in the coarse grid domain. MNWF computed results with $31 \%-44 \%$ fewer iterations than DC, 50\% fewer than DWF, and 2\%-25\% fewer than RNWF.

Meanwhile, for MCICSAM-W, HRIC, FBICS, and CUIBS, the advantage in speed of MNWF in comparison with the other techniques is less evident but existent. The MNWF

(b)

Fine grid $(9536 \mathrm{CV})$

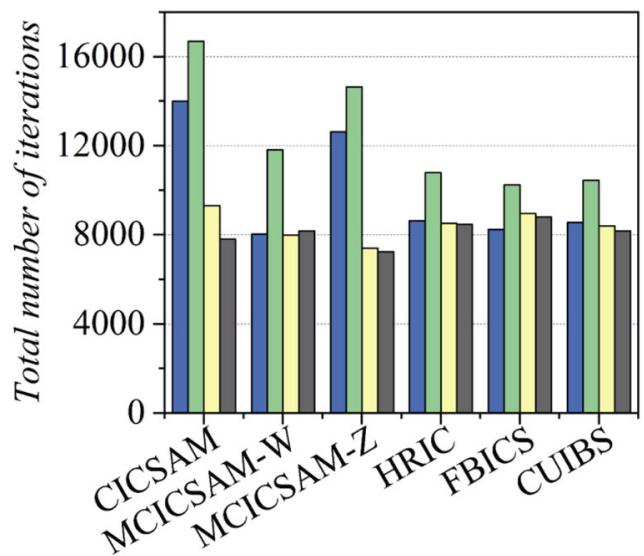

High-resolution schemes

Fig. 14 Total number of iterations required for the six HR schemes implemented using the DC, DWF, RNWF, and MNWF methods. The simulations of the dam-break flow impacting a rigid structure case were calculated for $0.9 \mathrm{~s}$. 

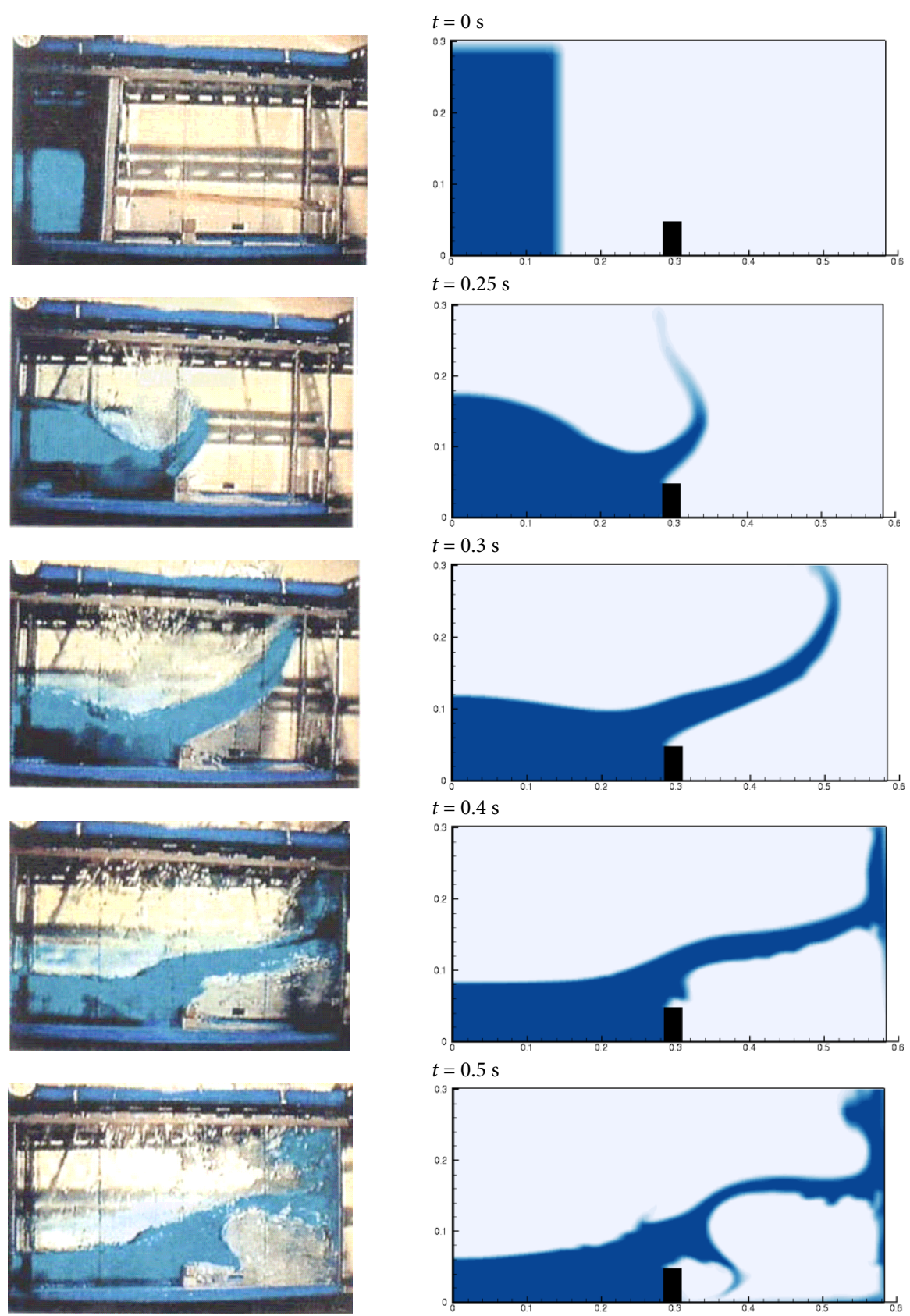

Fig. 15 A comparison of the free surface profile in the dam break experiment of Koshizuka (1995) at the same snapshot time. The results were obtained using the MCICSAM-Z scheme implemented with our new modified normalized weighting factor (MNWF) method.

is approximately $5 \%-7 \%$ faster than DC or RNWF, and $15 \%-32 \%$ than DWF. Furthermore, in this case, we noted that the numerical precision of the implementation method directly affects the calculation of the velocity. Thus, the velocity needs more interactions to converge, especially for DWF as this method on many occasions does not achieve the converge criterion.

Finally, in Fig. 14, a qualitative comparison between the simulation results obtained for the fine grid using the MCICSAM-Z scheme implemented with the proposed method MNWF and the experimental results from Koshizuka (1995) are presented to demonstrate the quality of the simulations.

\section{Conclusions}

We presented a new methodology, the MNWF method, to deal with the implementation of blended HR schemes in the context of multiphase flow. The method is based on the NWF methodology and produces a system of tridiagonal equations. The resulting coefficient matrix is always diagonally dominant, with $A_{P}$ coefficients greater than zero, giving a numerically stable method without the necessity of under 
relaxation. The main advantage of the new approach is its high rate of convergence in comparison to the other methods studied in this work, the DC, DWF, and RNWF methods. Also, MNWF does not degenerate the interface.

The superiority in the rate of convergence of the new MNWF compared to the other methods depends on the test case. However, generally, it can be concluded that the MNWF is about $5 \%-25 \%$ faster than the DC and RNWF methods, and markedly quicker than the DWF method. For medium and high Co cases, this difference exceeded $80 \%$ because the DWF method in many occasions does not converge, and the maximum number of iterations is executed. Thus, confirming what was mentioned by Darwish and Moukalled (1996), that the coefficients generated with the DWF form a numerically unstable system of equations.

Additionally, we observed that the convergence rate is strongly influenced by the methodology used to implement the blended HR schemes for medium and high local Co. Especially in the case of CICSAM and its modifications, which were initially developed to be implemented with the DWF method.

Also, observing the number of iterations for the three refined grids in the $2 \mathrm{D}$ rising bubble and in the dam break flow impacting a rigid structure test cases, we can conclude that the methodology is independent of the grid size, and the most influential parameter is the Courant number.

On the other hand, the accuracy of the results does not seem to be affected by the methodology used to implement the schemes numerically. Instead, it seems to be a characteristic of each scheme. Except for CICSAM, which generates less accurate results if it is implemented with RNWF.

In summary, the new MNWF methodology is highly recommended to work with medium and high Co as well as to improve the stability of the blended HR schemes.

\section{Acknowledgements}

This work is supported by the "Excellence Initiative" of the German Federal and State Governments within the Graduate School of Computational Engineering at Technische Universität Darmstadt.

\section{Funding note}

Open access funding provided by Projekt DEAL.

\section{References}

Chourushi, T. 2018. Computationally inexpensive and revised normalized weighting factor method for segregated solvers. Int J Comput Math, 95: 1622-1653.

Darwish, M. S., Moukalled, F. 1996. The normalized weighting factor method: A novel technique for accelerating the convergence of high-resolution convective schemes. Numer Heat Tr B: Fund, 30: 217-237.

Darwish, M., Moukalled, F. 2006. Convective schemes for capturing interfaces of free-surface flows on unstructured grids. Numer Heat $\operatorname{Tr}$ B: Fund, 49: 19-42.

Ferziger, J. H., Perić, M. 2012. Computational Methods for Fluid Dynamics, 3rd edn. Springer-Verlag Berlin Heidelberg.

Gaskell, P. H., Lau, A. K. C. 1988. Curvature-compensated convective transport: SMART, a new boundedness-preserving transport algorithm. Int J Numer Meth Fl, 8: 617-641.

Hirt, C. W., Nichols, B. D. 1981. Volume of fluid (VOF) method for the dynamics of free boundaries. J Comput Physs, 39: 201225.

Hogg, P. W., Gu, X. J., Emerson, D. R. 2006. An implicit algorithm for capturing sharp fluid interfaces in the volume of fluid advection method. In: Proceedings of the European Conference on Computational Fluid Dynamics.

Hysing, S., Turek, S., Kuzmin, D., Parolini, N., Burman, E., Ganesan, S., Tobiska, L. 2009. Quantitative benchmark computations of two-dimensional bubble dynamics. Int J Numer Meth Fl, 60: 1259-1288.

Koshizuka, S. 1995. A particle method for incompressible viscous flow with fluid fragmentation. Comput Fluid Dyn J, 4(29).

Leonard, B. P. 1991. The ULTIMATE conservative difference scheme applied to unsteady one-dimensional advection. Comput Method Appl M, 88: 17-74.

Leonard, B. P., Mokhtari, S. 1990. Beyond first-order upwinding: The ultra-sharp alternative for non-oscillatory steady-state simulation of convection. Int J Numer Meth Eng, 30: 729-766.

Malik, M., Fan, E. S. C., Bussmann, M. 2007. Adaptive VOF with curvature-based refinement. Int J Numer Meth Fl, 55: 693-712.

Meyer, J., Renzsch, H., Graf, K., Slawing, T. 2016. Advanced CDF-simulations of free-surface flows around modern sailing yachts using a newly developed OpenFOAM solver. In: Proceedings of the 22nd Chesapeake Sailing Yacht Symposium.

Moukalled, F., Mangani, L., Darwish, M. 2016. The Finite Volume Method in Computational Fluid Dynamics. Springer International Publishing Switzerland.

Muzaferija, S., Perić, M., Sames, P. C., Shellin, T. 1998. A two-fluid Navier-Stokes solver to simulate water entry. In: Proceedings of the 22nd Symposium on Naval Hydrodynamics, 638-651.

Patel, J. K., Natarajan, G. 2015. A generic framework for design of interface capturing schemes for multi-fluid flows. Comput Fluids, 106: 108-118.

Rubin, S. G., Khosla, P. K. 1977. Polynomial interpolation methods for viscous flow calculations. J Comput Phys, 24: 217-244.

Rudman, M. 1997. Volume-tracking methods for interfacial flow calculations. Int J Numer Meth Fl, 24: 671-691.

Sauer, J. 2000. Instationär kavitierende Strömungen: ein neues Modell, basierend auf front capturing $(\mathrm{VoF})$ und Blasendynamik. Universität Karlsruhe.

Tryggvason, G., Scardovelli, R., Zaleski, S. 2001. Direct Numerical Simulations of Gas-Liquid Multiphase Flows. Cambridge University Press. 
Tsui, Y. Y., Lin, S., Cheng, T., Wu, T. C. 2009. Flux-blending schemes for interface capture in two-fluid flows. Int J Heat Mass Tran, 52: $5547-5556$

Turek, S., Mierka, O., Bäumler, K. 2019. Numerical benchmarking for 3D multiphase flow: New results for a rising bubble. In: Numerical Mathematics and Advanced Applications ENUMATH 2017. Lecture Notes in Computational Science and Engineering, Vol. 126. Radu, F., Kumar, K., Berre, I., Nordbotten, J., Pop, I. Eds. Springer Cham, 593-601.

Ubbink, O. 1997. Numerical prediction of two fluid systems with sharp interfaces. Imperial College of Science, Technology \& Medicine, London, UK.

Ubbink, O., Issa, R. I. 1999. A method for capturing sharp fluid interfaces on arbitrary meshes. J Comput Phys, 153: 26-50.

Wackers, J., Koren, B., Raven, H. C., van der Ploeg, A., Starke, A. R., Deng, G. B., Queutey, P., Visonneau, M., Hino, T., Ohashi, K. 2011. Free-surface viscous flow solution methods for ship hydrodynamics. Arch Comput Method E, 18: 1-41.

Wacławczyk, T., Caner Gemici, Ö., Schäfer, M. 2007. Novel highresolution scheme for interface capturing in multi-phase flow. In: Proceedings of the 6th International Conference on Multiphase Flow, S1-Fri-A64.
Zalesak, S. T. 1979. Fully multidimensional flux-corrected transport algorithms for fluids. J Comput Phys, 31: 335-362.

Zhang, D., Jiang, C., Liang, D., Chen, Z., Yang, Y., Shi, Y. 2014. A refined volume-of-fluid algorithm for capturing sharp fluid interfaces on arbitrary meshes. J Comput Phys, 274: 709-736.

Open Access This article is licensed under a Creative Commons Attribution 4.0 International License, which permits use, sharing, adaptation, distribution and reproduction in any medium or format, as long as you give appropriate credit to the original author(s) and the source, provide a link to the Creative Commons licence, and indicate if changes were made.

The images or other third party material in this article are included in the article's Creative Commons licence, unless indicated otherwise in a credit line to the material. If material is not included in the article's Creative Commons licence and your intended use is not permitted by statutory regulation or exceeds the permitted use, you will need to obtain permission directly from the copyright holder.

To view a copy of this licence, visit http://creativecommons.org/ licenses/by/4.0/. 